\title{
DERIVING PLASMA DENSITIES AND ELEMENTAL ABUNDANCES FROM SERTS DIFFERENTIAL EMISSION MEASURE ANALYSIS
}

\author{
J. T. Schmelz ${ }^{1}$, J. A. Kimble ${ }^{1}$, AND J. L. R. SABA ${ }^{2,3}$ \\ ${ }^{1}$ Physics Department, University of Memphis, Memphis, TN 38152, USA; jschmelz@memphis.edu \\ ${ }^{2}$ Astronomy Department, University of Maryland, College Park, MD 20742, USA \\ ${ }^{3}$ NASA/Goddard Space Flight Center, Greenbelt, MD 20771, USA \\ Received 2012 June 7; accepted 2012 July 24; published 2012 August 30
}

\begin{abstract}
We use high-resolution spectral emission line data obtained by the SERTS instrument during three rocket flights to demonstrate a new approach for constraining electron densities of solar active region plasma. We apply differential emission measure (DEM) forward-fitting techniques to characterize the multithermal solar plasma producing the observed EUV spectra, with constraints on the high-temperature plasma from the Yohkoh Soft X-ray Telescope. In this iterative process, we compare line intensities predicted by an input source distribution to observed line intensities for multiple iron ion species, and search a broad range of densities to optimize $\chi^{2}$ simultaneously for the many available density-sensitive lines. This produces a density weighted by the DEM, which appears to be useful for characterizing the bulk of the emitting plasma over a significant range of temperature. This "DEM-weighted density" technique is complementary to the use of density-sensitive line ratios and less affected by uncertainties in atomic data and ionization fraction for any specific line. Once the DEM shape and the DEM-weighted density have been established from the iron lines, the relative elemental abundances can be determined for other lines in the spectrum. We have also identified spectral lines in the SERTS wavelength range that may be problematic.
\end{abstract}

Key words: Sun: abundances - Sun: corona - Sun: fundamental parameters - Sun: UV radiation - Sun: X-rays, gamma rays

Online-only material: color figures

\section{INTRODUCTION}

Plasma temperatures, densities, and elemental abundances are crucial parameters required to understand and model the solar corona. Despite the fact that differential emission measure (DEM) modeling has been available since the 1960s, most temperature measurements have been made under the assumption that the emitting plasma was isothermal. Recent results, however, imply that this simplification may not apply to as many coronal structures as we once thought. Densities, $n_{e}$, are usually found from the ratio of intensities of two spectral lines of the same ionization stage of a given element. When an appropriate pair of lines is not available, however, densities are sometimes estimated from observations of the emission measure, $\mathrm{EM}=n_{e}^{2} d l$, where the emitting length $(l)$ is determined from an inspection of the image and an assumption about the filling factor. Elemental abundances affect cooling timescales, energetics, pressure balance, and also determine the shape of the radiative loss function. They are tracers of the origins of the solar system, and have been used to track various components of the solar wind, solar energetic particles (SEPs), and coronal mass ejections back to the solar source.

Spectroscopic (Schmelz et al. 2012), SEP (Reames 1995), and solar wind (von Steiger et al. 2000, 2010) data suggest that the coronal-to-photospheric abundance ratios of elements with low first ionization potential (FIP $<10 \mathrm{eV}$ ) seem to be enhanced relative to those with high FIP $(>11 \mathrm{eV})$. This fractionation probably results from a separation of ions and neutrals. Two different empirical models have been suggested in the literature: (1) low-FIP elements may be enhanced by about a factor of four with respect to their photospheric values while high-FIP elements are the same in the corona and the photosphere (Feldman 1992), or (2) low-FIP elements may be the same in the corona and the photosphere while high-FIP elements are depleted by about a factor of four with respect to their photospheric values (Meyer 1985). However, many of the elemental abundance measurements made in the 1980s and 1990s and used to support one or the other of these models were derived with old atomic data and under the assumption that the coronal plasma was isothermal.

In this paper, we take advantage of the improved understanding of the thermal structure of the corona, better multithermal analysis techniques, and most importantly, updated atomic modeling and ionization balance calculations for coronal emission lines. The spectral data from three different active regions observed during the 1993, 1995, and 1997 flights of the Goddard Solar EUV Rocket Telescope and Spectrograph (SERTS-93, SERTS-95, and SERTS-97) were described in detail by Brosius et al. (1996, 1998, 2000). We have used these spectra along with updated atomic physics data from CHIANTI v.7 (Dere et al. 1997; Landi et al. 2012) to derive the DEM for the observed active region plasma. Broadband X-ray filter data from the Yohkoh Soft X-ray Telescope (SXT) were used to constrain the high-temperature end of these DEM distributions. These results were used to derive a DEM-weighted plasma density and to test the new set of "recommended coronal" elemental abundances proposed by Schmelz et al. (2012), an update of the "hybrid" abundances of Fludra \& Schmelz (1999).

\section{OBSERVATIONS}

The SERTS (Neupert et al. 1992) payloads were launched on Terrier-boosted Black Brant rockets from White Sands, New Mexico. Each incorporated a grazing incidence Wolter 2 telescope, which formed a real image of the Sun on the entrance aperture of a quasi-stigmatic spectrograph. The aperture allowed 
spectra to be obtained along a long narrow slit which connected two rectangular lobes, within which spectroheliograms were imaged. In our analysis, SERTS images were used only for coregistration of the SERTS and SXT data; otherwise, only the SERTS slit spectra of the active region targets were considered.

In each of the three flights (see Brosius et al. 1996, 1998, 2000 for details), the instrument included a multilayer-coated toroidal diffraction grating which enhanced the instrument sensitivity over a portion of the spectrum as compared with that of a standard gold grating (such as that used in the 1989 SERTS flight). SERTS-93 and SERTS-97 used the same multilayercoated grating, which enhanced the first-order response around $300 \AA$ by up to a factor of nine over a gold grating. SERTS-95 used a different grating, which enhanced the sensitivity of the second-order wave band (171-225 $\AA$ ), producing greatest sensitivity in the range $\sim 182-208 \AA$.

SERTS-93 was launched on 1993 August 17 at 1800 UT and recorded spectrographic data between 100 and $492 \mathrm{~s}$ after launch. Spectra were obtained from 235 to $450 \AA$; the measured instrumental FWHM resolution varied in a known way along the slit and was typically $\sim 55 \mathrm{~m} \AA$; spatial resolution was $\sim 5$ arcsec. An active region spectrum with 65 prominent lines was obtained from an average of the 163 arcsec portion of the slit that bisected Active Region (AR) 7563 (at heliographic coordinates S01 W15); the slit was roughly parallel to the direction of the loops.

SERTS-95 was launched on 1995 May 15 at 1800 UT and recorded spectrographic data for nearly 7 minutes. The FWHM spectral resolution was $\sim 30 \mathrm{~m} \AA$ in second order $(171-225 \AA$ ) and $\sim 55 \mathrm{~m} \AA$ in first order $(235-335 \AA$ ); the spatial resolution was $\sim 5$ arcsec. A spectrum was obtained for AR 7870 (at N09 W22), by averaging the spectra over a 226 arcsec portion of the slit that bisected the region; in this case, the slit was approximately perpendicular to the direction of the loops and crossed near the loop tops. Over 100 lines were identified in the spectrum.

SERTS-97 was launched on 1997 November 18 at 1935 UT and recorded spectrographic data for nearly $400 \mathrm{~s}$. The FWHM spectral resolution was $115 \mathrm{~m} \AA$; the spatial resolution was $\sim 7$ arcsec. A spectrum was obtained for AR 8108 (at N21 E18) by averaging over a 221 arcsec segment of the slit covering the region. The spectrum included 95 emission lines-52 identified spectral lines plus 43 weaker features that correspond to blends or have uncertain identification.

The SXT images are taken in broadband soft X-rays (approximately 2-60 ̊). Details of the instrument and operation modes can be obtained from the paper by Tsuneta et al. (1991); the Yohkoh Analysis Guide (www.lmsal.com/SXT/) documents much of the available software for data reduction and analysis. Also, please see the Yohkoh Legacy data Archive (ylstone.physics.montana.edu/ylegacy/). SXT provided cotemporal high-temperature $(>2 \mathrm{MK})$ images of the targeted active regions. The data were co-registered by the SERTS team. For SERTS-93, they used partial-frame, high-resolution (2.455 arcsec pixel) images in three filters: thin Al (379.95 $\left.\mathrm{DN} \mathrm{s}^{-1}\right), \operatorname{AlMgMn}\left(192.81 \mathrm{DN} \mathrm{s}^{-1}\right)$, and thick $\mathrm{Al}\left(9.00 \mathrm{DN} \mathrm{s}^{-1}\right)$. For SERTS-95, they used full-frame images with 4.91 arcsec pixels in two filters: thin $\mathrm{Al}\left(2968.10 \mathrm{DN} \mathrm{s}^{-1}\right)$ and AlMgMn (15.73.45 $\left.\mathrm{DN} \mathrm{s}^{-1}\right)$. And for SERTS-97, they used full-frame images in the AlMgMn filter (821.57 $\mathrm{DN} \mathrm{s}^{-1}$ ).

\section{ANALYSIS}

In this paper, we analyze active region data obtained simultaneously by SERTS and SXT. The SERTS iron lines used in this analysis are listed in Table 1 and the non-iron lines are listed in Table 2. These data, including the line identifications and wavelengths, are from the original SERTS papers by Brosius et al. $(1996,1998,2000)$. If a line appeared in more than one data set, we listed the wavelength from Brosius et al. (2000). The uncertainty of the line centroid is typically less than $10 \mathrm{~m} \AA$ for most lines (Brosius et al. 1996). We have also listed the wavelengths from CHIANTI v.7 for comparison. By themselves, the available SERTS data do not constrain higher temperature active region plasma. The SERTS wavelength range does include higher temperature flare lines, but none of them were detected for these active regions, and the signal-to-noise level was not high enough to use the absence of the lines as a significant hightemperature constraint. Combining SXT data with the SERTS data provides the constraint on the high-temperature end of the DEM curve which is typically poorly defined in studies using EUV data alone.

Although the majority of the SERTS emission lines have significantly cooler temperature response than that of the dominant SXT response, there is sufficient overlap of temperature sensitivity to permit consistency checks between the SERTS and SXT analyses. So, the combined data set is able to yield a reliable multithermal analysis over the range (1-10 MK) needed to adequately characterize the thermal distribution of coronal plasma in the observed active regions.

The SXT response functions are computed by folding a synthetic solar spectrum through the measured effective area for each of the five different filters. Standard SXT analysis was done with the ionization balance calculations of Arnaud \& Rothenflug (1985) and Meyer (1985) coronal abundances. Standard SERTS analysis, however, used Arnaud \& Raymond (1992) ionic fractions for iron and Feldman (1992) abundances.

Schmelz et al. (1999) showed that the choice of abundance normalization and/or ionization fraction used in the SXT analysis can severely affect models of the coronal plasma that require the temperature or emission measure distribution. Given the sensitivity of the SXT filter responses to the change in elemental abundance normalization and iron ionization fractions, joint use of the SXT and SERTS data requires that the SXT response functions and the SERTS contribution functions be generated with the same set of elemental abundances and ionization fractions. For the multithermal analysis presented in this paper, we adopted the ionization fractions of Bryans et al. (2009), which the CHIANTI team has recalculated with a temperature resolution of $\log T=0.05$. For our initial best guess of the elemental abundances, we have adopted the recommended coronal abundance set of Schmelz et al. (2012).

\subsection{Method}

We use two different DEM methods in our multithermal analysis. The first, DEM_manual (Schmelz et al. 2011a, 2011b), is a forward-fitting technique with a manual manipulation of the DEM. The best fit is determined from a $\chi^{2}$ minimization of the differences between the observed and predicted line fluxes. The main advantages of this method are that no smoothing is required beyond that imposed by the temperature resolution of the spectral line contribution functions or SXT response functions. Also, no a priori shape (Gaussian or double Gaussian, for example) is imposed on the final DEM curve. The main disadvantage of DEM_manual is that it is not usually possible to explore a broad parameter space, so families of solutions might be missed. 
Table 1

SERTS Iron Line Intensities (erg $\mathrm{cm}^{-2} \mathrm{~s}^{-1} \mathrm{sr}^{-1}$ )

\begin{tabular}{|c|c|c|c|c|c|c|c|c|}
\hline & Ion & $\log T$ & $\begin{array}{c}\text { SERTS } \\
\lambda(\AA)\end{array}$ & $\begin{array}{c}\text { CHIANTI } \\
\lambda(\AA)\end{array}$ & SERTS-93 & SERTS-95 & SERTS-97 & Notes \\
\hline 1 & Fe VIII & 5.70 & 185.221 & 185.2133 & $\ldots$ & $305 \pm 42.3$ & $\ldots$ & $\mathrm{a}$ \\
\hline 2 & Fe VIII & 5.70 & 187.247 & 187.2407 & $\ldots$ & $15.6 \pm 7.40$ & $\ldots$ & $\mathrm{b}$ \\
\hline 3 & Fe IX & 5.90 & 171.074 & 171.0733 & $\ldots$ & $208 \pm 62.0$ & $\ldots$ & \\
\hline 4 & Fe IX & 5.90 & 218.943 & 218.9373 & $\ldots$ & $98.9 \pm 30.1$ & $\ldots$ & $\mathrm{c}$ \\
\hline 5 & Fe $x$ & 6.05 & 174.526 & 174.5310 & $\ldots$ & $654 \pm 83.0$ & $\ldots$ & \\
\hline 6 & Fe $x$ & 6.05 & 175.265 & 175.2630 & $\ldots$ & $135 \pm 32.4$ & $\ldots$ & \\
\hline 7 & $\mathrm{Fe} x$ & 6.05 & 177.240 & 177.2400 & $\ldots$ & $335 \pm 47.5$ & $\ldots$ & \\
\hline 8 & Fe $x$ & 6.05 & 184.534 & 184.5370 & $\ldots$ & $164 \pm 23.8$ & $\ldots$ & \\
\hline 9 & Fe $x$ & 6.05 & 190.046 & 190.0370 & $\ldots$ & $47.9 \pm 9.40$ & $\ldots$ & \\
\hline 10 & Fex & 6.05 & 345.735 & 345.7380 & $106 \pm 12.7$ & $\ldots$ & $57.0 \pm 9.60$ & \\
\hline 11 & Fe XI & 6.15 & 180.382 & 180.4012 & $\ldots$ & $3440 \pm 341$ & $\ldots$ & $\mathrm{a}$ \\
\hline 12 & Fe XI & 6.15 & 181.125 & 181.1302 & $\ldots$ & $123 \pm 35.6$ & $\ldots$ & \\
\hline 13 & Fe XI & 6.15 & 182.166 & 182.1672 & $\ldots$ & $237 \pm 33.4$ & $\ldots$ & \\
\hline 14 & Fe XI & 6.15 & 184.800 & 184.7930 & $\ldots$ & $31.5 \pm 14.0$ & $\ldots$ & $\mathrm{b}$ \\
\hline 15 & Fe XI & 6.15 & 188.214 & 188.2165 & $\ldots$ & $385 \pm 77.3$ & $\ldots$ & \\
\hline 16 & Fe XI & 6.15 & 188.295 & 188.2994 & $\ldots$ & $276 \pm 51.6$ & $\ldots$ & \\
\hline 17 & Fe XI & 6.15 & 189.185 & 189.1234 & $\ldots$ & $32.8 \pm 8.90$ & $\ldots$ & $\mathrm{c}$ \\
\hline 18 & Fe XI & 6.15 & 189.722 & 189.7112 & $\ldots$ & $26.4 \pm 6.70$ & $\ldots$ & \\
\hline 19 & Fe XI & 6.15 & 192.813 & 192.8137 & $\ldots$ & $68.3 \pm 9.80$ & $\ldots$ & \\
\hline 20 & Fe XI & 6.15 & 202.415 & 202.4242 & $\ldots$ & $73.7 \pm 17.3$ & $\ldots$ & $\mathrm{c}$ \\
\hline 21 & Fe XI & 6.10 & 308.548 & 308.5445 & $28.9 \pm 11.4$ & $120 \pm 37.4$ & $33.8 \pm 9.6$ & $b^{*}, c^{*}$ \\
\hline 22 & Fe XI & 6.15 & 308.991 & 308.9916 & $\ldots$ & $\ldots$ & $29.1 \pm 11.3$ & $\mathrm{~b}$ \\
\hline 23 & Fe XI & 6.10 & 341.129 & 341.1136 & $71.1 \pm 9.23$ & $\ldots$ & $37.9 \pm 6.60$ & $\mathrm{a}$ \\
\hline 24 & Fe XI & 6.10 & 349.032 & 349.0466 & $\ldots$ & $\ldots$ & $7.5 \pm 2.50$ & \\
\hline 25 & $\mathrm{Fe} \mathrm{XI}$ & 6.10 & 352.674 & 352.6709 & $177 \pm 20.2$ & $\ldots$ & $136 \pm 22.0$ & \\
\hline 26 & Fe XI & 6.10 & 356.553 & 356.5193 & $19.3 \pm 8.38$ & $\ldots$ & $\ldots$ & $\mathrm{b}$ \\
\hline 27 & Fe XI & 6.10 & 358.694 & 358.6138 & $60.0 \pm 11.1$ & $\ldots$ & $\ldots$ & a \\
\hline 28 & $\mathrm{Fe} \mathrm{XI}$ & 6.10 & 369.205 & 369.1635 & $60.0 \pm 13.2$ & $\ldots$ & $\ldots$ & \\
\hline \multirow[t]{2}{*}{29} & Fe XII & 6.20 & 186.867 & 186.8540 & $\ldots$ & $498 \pm 64.0$ & $\ldots$ & $\mathrm{c}$ \\
\hline & & & & 186.8870 & & & & \\
\hline 30 & Fe XII & 6.20 & 192.390 & 192.3940 & $\ldots$ & $206 \pm 25.1$ & $\ldots$ & \\
\hline 31 & Fe XII & 6.20 & 193.505 & 193.5090 & $\cdots$ & $487 \pm 55.4$ & $\cdots$ & \\
\hline 32 & Fe XII & 6.20 & 195.117 & 195.1190 & $\ldots$ & $869 \pm 100$ & $\ldots$ & \\
\hline 33 & Fe XII & 6.20 & 196.642 & 196.6400 & $\ldots$ & $120 \pm 18.0$ & $\ldots$ & \\
\hline 34 & Fe XII & 6.20 & 211.736 & 211.7320 & $\ldots$ & $94.2 \pm 29.1$ & $\ldots$ & \\
\hline 35 & Fe XII & 6.20 & 217.277 & 217.2760 & $\ldots$ & $97.7 \pm 35.3$ & $\ldots$ & $\mathrm{b}, \mathrm{c}$ \\
\hline 36 & Fe XII & 6.20 & 219.449 & 219.4370 & $\cdots$ & $73.5 \pm 22.5$ & $\ldots$ & \\
\hline 37 & Fe XII & 6.20 & 338.273 & 338.2630 & $92.6 \pm 11.3$ & $\ldots$ & $57.6 \pm 9.50$ & \\
\hline 38 & Fe XII & 6.20 & 346.849 & 346.8520 & $115 \pm 14.0$ & $\ldots$ & $77.9 \pm 12.8$ & \\
\hline 39 & Fe XII & 6.20 & 352.112 & 352.1060 & $190 \pm 22.1$ & $\ldots$ & $171 \pm 27.0$ & \\
\hline 40 & Fe XII & 6.20 & 364.494 & 364.4670 & $257 \pm 29.5$ & $\ldots$ & $\ldots$ & \\
\hline 41 & Fe XIII & 6.25 & 196.519 & 196.5253 & $\ldots$ & $135 \pm 33.4$ & $\ldots$ & \\
\hline 42 & Fe XIII & 6.25 & 200.017 & 200.0216 & $\ldots$ & $304 \pm 36.7$ & $\ldots$ & \\
\hline 43 & Fe XIII & 6.25 & 201.118 & 201.1259 & $\cdots$ & $470 \pm 60.6$ & $\cdots$ & \\
\hline 44 & Fe XIII & 6.25 & 202.042 & 202.0443 & $\ldots$ & $1210 \pm 138$ & $\ldots$ & \\
\hline 45 & Fe XIII & 6.25 & 203.164 & 203.1653 & $\ldots$ & $154 \pm 24.2$ & $\ldots$ & \\
\hline \multirow[t]{2}{*}{46} & Fe XIII & 6.25 & 203.820 & 203.7722 & $\ldots$ & $1930 \pm 238$ & $\ldots$ & \\
\hline & & & & 203.7957 & & & & \\
\hline 47 & Fe XIII & 6.25 & 204.255 & 204.2626 & $\ldots$ & $195 \pm 29.7$ & $\ldots$ & \\
\hline 48 & Fe XIII & 6.25 & 204.950 & 204.9422 & $\ldots$ & $267 \pm 44.5$ & $\ldots$ & $\mathrm{c}$ \\
\hline 49 & Fe XIII & 6.25 & 209.623 & 209.6199 & $\ldots$ & $210 \pm 33.1$ & $\ldots$ & \\
\hline 50 & Fe XIII & 6.25 & 209.908 & 209.9167 & $\ldots$ & $225 \pm 43.6$ & $\ldots$ & $\mathrm{c}$ \\
\hline 51 & Fe XIII & 6.25 & 213.764 & 213.7686 & $\ldots$ & $91.7 \pm 20.6$ & $\ldots$ & \\
\hline 52 & Fe XIII & 6.25 & 221.813 & 221.8281 & $\ldots$ & $218 \pm 32.5$ & $\ldots$ & \\
\hline 53 & Fe XIII & 6.25 & 240.687 & 240.6964 & $\ldots$ & $160 \pm 66.2$ & $\ldots$ & $a, b$ \\
\hline 54 & Fe XIII & 6.25 & 246.187 & 246.2095 & $\ldots$ & $246 \pm 47.4$ & $\ldots$ & \\
\hline 55 & Fe XIII & 6.25 & 251.939 & 251.9529 & $\cdots$ & $332 \pm 65$ & $\cdots$ & \\
\hline 56 & Fe XIII & 6.25 & 311.583 & 311.5475 & $\ldots$ & $\ldots$ & $16.4 \pm 7.40$ & $\mathrm{~b}$ \\
\hline 57 & Fe XIII & 6.25 & 312.172 & 312.1748 & $105 \pm 16.5$ & $172 \pm 33.8$ & $61.6 \pm 12.0$ & $c^{*}$ \\
\hline 58 & Fe XIII & 6.25 & 312.897 & 312.8683 & $36.8 \pm 9.13$ & .. & $33.5 \pm 7.90$ & \\
\hline 59 & Fe XIII & 6.25 & 318.125 & 318.1302 & $85.9 \pm 12.6$ & $112 \pm 42.8$ & $64.7 \pm 11.6$ & $b^{*}$ \\
\hline 60 & Fe XIII & 6.25 & 320.807 & 320.8010 & $170 \pm 19.8$ & $195 \pm 57.8$ & $118 \pm 19.0$ & \\
\hline 61 & Fe XIII & 6.25 & 321.475 & 321.4662 & $31.1 \pm 5.52$ & $\ldots$ & $28.7 \pm 6.00$ & \\
\hline 62 & Fe XIII & 6.25 & 348.179 & 348.1840 & $210 \pm 24.3$ & $\ldots$ & $170 \pm 27.0$ & $\mathrm{c}^{*}$ \\
\hline 63 & Fe XIII & 6.25 & 359.665 & 359.6445 & $170 \pm 19.7$ & $\ldots$ & $\ldots$ & \\
\hline
\end{tabular}


Table 1

(Continued)

\begin{tabular}{|c|c|c|c|c|c|c|c|c|}
\hline & Ion & $\log T$ & $\begin{array}{c}\text { SERTS } \\
\lambda(\AA)\end{array}$ & $\begin{array}{c}\text { CHIANTI } \\
\lambda(\AA)\end{array}$ & SERTS-93 & SERTS-95 & SERTS-97 & Notes \\
\hline 64 & Fe XIII & 6.25 & 359.852 & 359.8399 & $41.7 \pm 5.81$ & $\ldots$ & $\ldots$ & \\
\hline 65 & Fe XIV & 6.30 & 211.317 & 211.3172 & $\ldots$ & $2510 \pm 287$ & $\ldots$ & \\
\hline 66 & Fe XIV & 6.30 & 219.121 & 219.1305 & $\ldots$ & $748 \pm 89.1$ & $\ldots$ & $\mathrm{d}$ \\
\hline 67 & Fe XIV & 6.30 & 220.090 & 220.0849 & $\ldots$ & $621 \pm 84.2$ & $\ldots$ & \\
\hline 68 & Fe XIV & 6.30 & 252.168 & 252.1996 & $\ldots$ & $364 \pm 73.3$ & $\ldots$ & \\
\hline 70 & Fe XIV & 6.30 & 264.768 & 264.7889 & $\ldots$ & $1270 \pm 156$ & $\ldots$ & \\
\hline 71 & Fe XIV & 6.30 & 270.511 & 270.5208 & $\ldots$ & $674 \pm 118$ & $\ldots$ & \\
\hline 72 & Fe XIV & 6.30 & 274.193 & 274.2037 & $1140 \pm 167$ & $1360 \pm 176$ & $\ldots$ & $\mathrm{c}$ \\
\hline 73 & Fe XIV & 6.30 & 289.140 & 289.1508 & $60.5 \pm 21.6$ & $213 \pm 46.4$ & $\ldots$ & $b^{*}, c^{*}$ \\
\hline 74 & Fe XIV & 6.30 & 334.173 & 334.1783 & $645 \pm 72.9$ & $923 \pm 107$ & $554 \pm 89.0$ & $c^{*}$ \\
\hline 75 & Fe XIV & 6.30 & 353.856 & 353.8364 & $291 \pm 32.9$ & $\ldots$ & $\ldots$ & \\
\hline 76 & $\mathrm{Fe} x \mathrm{~V}$ & 6.35 & 243.771 & 243.7940 & $\ldots$ & $668 \pm 102$ & $\ldots$ & \\
\hline 78 & $\mathrm{Fe} x \mathrm{v}$ & 6.35 & 302.322 & 302.3340 & $\ldots$ & $\ldots$ & $37.3 \pm 10.3$ & $\mathrm{a}$ \\
\hline 79 & $\mathrm{Fe} x \mathrm{~V}$ & 6.35 & 304.873 & 304.8940 & $\ldots$ & $\ldots$ & $102 \pm 18.0$ & \\
\hline 80 & $\mathrm{Fe} x \mathrm{~V}$ & 6.35 & 307.764 & 307.7470 & $\ldots$ & $\ldots$ & $43.1 \pm 11.1$ & \\
\hline 81 & $\mathrm{Fe} x \mathrm{v}$ & 6.35 & 312.566 & 312.5590 & $41.4 \pm 9.02$ & $\ldots$ & $51.7 \pm 10.5$ & $\mathrm{a}$ \\
\hline 82 & $\mathrm{Fe} X \mathrm{~V}$ & 6.35 & 317.640 & $\ldots$ & $\ldots$ & $\ldots$ & $3.60 \pm 3.10$ & $\mathrm{~b}$ \\
\hline 83 & $\mathrm{Fe} x \mathrm{~V}$ & 6.35 & 321.787 & 321.7690 & $16.3 \pm 3.64$ & $\ldots$ & $23.4 \pm 5.30$ & \\
\hline 84 & $\mathrm{Fe} x \mathrm{v}$ & 6.35 & 324.975 & 324.9750 & $\ldots$ & $\ldots$ & $7.80 \pm 3.60$ & $\mathrm{~b}$ \\
\hline 85 & $\mathrm{Fe} X \mathrm{~V}$ & 6.35 & 327.032 & 327.0330 & $52.2 \pm 6.64$ & $\ldots$ & $74.4 \pm 12.1$ & \\
\hline 86 & $\mathrm{Fe} x \mathrm{~V}$ & 6.35 & 330.994 & $\ldots$ & $\ldots$ & $\ldots$ & $6.20 \pm 3.60$ & $\mathrm{~b}$ \\
\hline 87 & $\mathrm{Fe} x \mathrm{~V}$ & 6.35 & 417.296 & 417.2580 & $241 \pm 29.5$ & $\ldots$ & $\ldots$ & \\
\hline 88 & Fe XVI & 6.45 & 251.053 & 251.0630 & $\ldots$ & $521 \pm 74.6$ & $\ldots$ & \\
\hline 89 & Fe XVI & 6.45 & 262.965 & 262.9760 & $\ldots$ & $858 \pm 104$ & $\ldots$ & \\
\hline 90 & Fe XVI & 6.45 & 335.404 & 335.4100 & $4090 \pm 470$ & $12000 \pm 1350$ & $5020 \pm 804$ & \\
\hline 91 & Fe XVI & 6.45 & 360.782 & 360.7590 & $2040 \pm 232$ & $\ldots$ & $\ldots$ & \\
\hline 92 & Fe XVII & 6.70 & 347.848 & 347.8170 & $9.17 \pm 3.73$ & $\ldots$ & $17.2 \pm 6.30$ & $\mathrm{~b}, \mathrm{c}$ \\
\hline
\end{tabular}

Notes.

${ }^{\text {a }}$ CHIANTI indicates that these lines are blended; they are not used in our analysis.

$\mathrm{b}$ These detections are $<3 \sigma ;{ }^{*}$ indicates only one data set value is problematic.

${ }^{\mathrm{c}}$ Predicted intensity is $>5 \sigma$ low compared with observed; * indicates only SERTS-95 value is problematic.

${ }^{\mathrm{d}}$ Predicted intensity is $>5 \sigma$ high compared with observed.

Our second DEM method is xrt_dem_iterative2, a program first developed (Weber et al. 2004) and tested (Schmelz et al. 2009) for XRT data alone, but now applied more generally (see, e.g., Schmelz et al. 2010; Winebarger et al. 2011). The routine employs a forward-fitting approach where a DEM is guessed and folded through each response to generate predicted fluxes. This process is iterated to minimize $\chi^{2}$ for the predicted-to-observed flux ratios. The DEM function is interpolated using $N_{i}-1$ splines, representing the degrees of freedom for $N_{i}$ observations. This routine uses Monte Carlo iterations to estimate errors on the DEM solution. For each iteration, the observed flux in each line/filter was varied randomly and the program was run again with the new values. The distribution of these variations was Gaussian with a centroid equal to the observed flux and a width equal to the uncertainty.

The intensity for each spectral line observed by SERTS is proportional to $\sum A \times G(T) \times \operatorname{DEM}(T) \times \Delta T$, where $A$ is the elemental abundance, $G(T)$ is the contribution function $\left(\mathrm{erg} \mathrm{cm} \mathrm{cm}^{3} \mathrm{~s}^{-1}\right)$ from CHIANTI, and DEM is the differential emission measure $\left(\mathrm{cm}^{-5} \mathrm{~K}^{-1}\right)$. The intensity for each SXT filter is proportional to $\sum \operatorname{Resp}(T) \times \operatorname{DEM}(T) \Delta T$, where $\operatorname{Resp}(T)$ is the response function (DN s ${ }^{-1}$ pixel $^{-1}$ per unit emission measure) calculated from the instrument effective areas available in SolarSoft and the CHIANTI synthetic solar spectrum with the same abundance and ionization equilibrium assumptions used for the spectral lines. Note that the SERTS and SXT data do not have to be converted to the same units, as long as the proper contribution and response functions are used.

The DEM curves in Figure 1 were constructed using only the SERTS iron lines (Table 1) and the SXT broadband filter data, which depend strongly on the strengths of the iron lines in the synthetic spectrum that is folded through the effective area of each filter. These curves were constructed with DEM_manual. Figure 2 shows the comparison of the DEM_manual curve in black and the Monte Carlos from xrt_dem_iterative 2 in red. This plot is made with the SERTS-97 data set only, but the results apply more generally to SERTS-93 and SERTS-95. Both the agreement between the different methods and the tightness of the Monte Carlos are encouraging, showing that the DEM shape is indeed determined by the data. Integrating over the coronal temperature range for the curves in Figure 1, we find that the total column emission measure is EM $=1.2 \times 10^{28} \mathrm{~cm}^{-5}$ for SERTS-93, EM = $2.8 \times 10^{28} \mathrm{~cm}^{-5}$ for SERTS-95, and $\mathrm{EM}=1.5 \times 10^{28} \mathrm{~cm}^{-5}$ for SERTS-97.

Figure 3 shows results for the SERTS-95 data using xrt_dem_iterative2. The DEM in the top panel uses the SERTS iron lines only (without SXT). The Monte Carlos begin to deviate above $\log T=6.6$, indicating that the DEM is not well constrained by the data. The bottom panel shows the improvement when SXT data are included in the analysis. The distribution 
Table 2

SERTS Non-iron Line Intensities (erg $\mathrm{cm}^{-2} \mathrm{~s}^{-1} \mathrm{sr}^{-1}$ )

\begin{tabular}{|c|c|c|c|c|c|c|c|c|}
\hline & Ion & $\log T$ & $\begin{array}{c}\text { SERTS } \\
\lambda(\AA)\end{array}$ & $\begin{array}{c}\text { CHIANTI } \\
\lambda(\AA)\end{array}$ & SERTS-93 & SERTS-95 & SERTS-97 & Notes \\
\hline 1 & $\mathrm{Mg} \mathrm{V}$ & 5.50 & 351.114 & 351.0850 & $\ldots$ & $\ldots$ & $16.7 \pm 4.90$ & \\
\hline 2 & $\mathrm{Mg}$ VI & 5.65 & 349.149 & $\begin{array}{l}349.1249 \\
349.1639\end{array}$ & $80.3 \pm 15.1$ & $\ldots$ & $55.9 \pm 10.5$ & \\
\hline 3 & Mg VII & 5.80 & 319.026 & 319.0340 & $51.9 \pm 8.2$ & $\ldots$ & $55.9 \pm 10.5$ & \\
\hline 4 & Mg VII & 5.80 & 367.698 & $\begin{array}{l}367.6780 \\
367.6880\end{array}$ & $49.5 \pm 11.1$ & $\ldots$ & $\ldots$ & \\
\hline 5 & Mg VIII & 5.90 & 311.769 & 311.7730 & $39.5 \pm 7.85$ & $\ldots$ & $20.9 \pm 6.00$ & \\
\hline 6 & Mg VIII & 5.90 & 313.734 & 313.7440 & $67.8 \pm 10.5$ & $\ldots$ & $71.5 \pm 13.4$ & \\
\hline 7 & Mg VIII & 5.90 & 315.022 & 315.0160 & $232 \pm 27.0$ & $144 \pm 46.4$ & $183 \pm 30.0$ & \\
\hline 8 & Mg VIII & 5.90 & 317.017 & 317.0280 & $55.9 \pm 10.2$ & $62.1 \pm 25.7$ & $34.8 \pm 8.40$ & $a^{*}$ \\
\hline 9 & Mg VIII & 5.90 & 338.997 & 338.9840 & $64.2 \pm 8.48$ & $\ldots$ & $29.7 \pm 5.40$ & \\
\hline 10 & Mg IX & 6.00 & 368.093 & 368.0713 & $943 \pm 110$ & $\ldots$ & $\ldots$ & \\
\hline 11 & $\mathrm{Al} \mathrm{x}$ & 6.10 & 332.784 & 332.7900 & $106 \pm 12.7$ & $173 \pm 44.1$ & $112 \pm 18.0$ & \\
\hline 12 & Si VIII & 5.95 & 214.757 & 214.7590 & $\ldots$ & $102 \pm 37.6$ & $\ldots$ & $\mathrm{b}, \mathrm{c}$ \\
\hline 13 & Si VIII & 5.95 & 314.358 & 314.3560 & $56.0 \pm 10.2$ & $\ldots$ & $36.8 \pm 8.50$ & \\
\hline 14 & Si VIII & 5.95 & 316.208 & 316.2180 & $112 \pm 17.4$ & $\ldots$ & $71.1 \pm 12.8$ & \\
\hline 15 & Si VIII & 5.95 & 319.841 & 319.8400 & $148 \pm 18.4$ & $\ldots$ & $91.2 \pm 15.3$ & \\
\hline 16 & Si IX & 6.05 & 258.077 & 258.0823 & $\ldots$ & $170 \pm 49.2$ & $\ldots$ & $\mathrm{b}$ \\
\hline 17 & Si IX & 6.05 & 292.756 & 292.7594 & $\ldots$ & $126 \pm 49.3$ & $\ldots$ & $\mathrm{a}, \mathrm{b}$ \\
\hline 18 & Si IX & 6.05 & 292.858 & $\begin{array}{l}292.8092 \\
292.8546\end{array}$ & $\ldots$ & $61.6 \pm 44.2$ & $\ldots$ & $\mathrm{a}, \mathrm{b}$ \\
\hline 19 & Si IX & 6.05 & 296.108 & 296.1135 & $270 \pm 41.0$ & $\ldots$ & $\ldots$ & \\
\hline 20 & Si IX & 6.05 & 341.971 & 341.9511 & $44.3 \pm 6.25$ & $\ldots$ & $28.3 \pm 5.20$ & \\
\hline 21 & Si IX & 6.05 & 344.987 & 344.9543 & $\ldots$ & $\ldots$ & $16.7 \pm 4.10$ & \\
\hline 22 & Si IX & 6.05 & 345.138 & 345.1210 & $124 \pm 15.5$ & $\ldots$ & $65.7 \pm 10.9$ & \\
\hline 23 & Si IX & 6.05 & 349.871 & $\begin{array}{l}349.7917 \\
349.8602\end{array}$ & $197 \pm 23.2$ & $\ldots$ & $121 \pm 20.0$ & \\
\hline 24 & $\mathrm{Six}$ & 6.15 & 258.361 & 258.3742 & $\ldots$ & $379 \pm 71.8$ & $\ldots$ & \\
\hline 25 & Si IX & 6.15 & 261.043 & 261.0567 & $\ldots$ & $265 \pm 66.9$ & $\ldots$ & c \\
\hline 26 & Si IX & 6.15 & 347.401 & 347.4026 & $286 \pm 32.6$ & $\ldots$ & $187 \pm 30.0$ & \\
\hline 27 & Si IX & 6.15 & 356.052 & $\begin{array}{l}356.0381 \\
356.0496\end{array}$ & $255 \pm 30.0$ & $\ldots$ & $\ldots$ & \\
\hline 28 & Si XI & 6.20 & 303.323 & 303.3268 & $2880 \pm 326$ & $3360 \pm 386$ & $2340 \pm 370$ & $\mathrm{c}^{*}$ \\
\hline 29 & Si XI & 6.20 & 365.431 & 365.4390 & $65.6 \pm 11.1$ & $\ldots$ & $\ldots$ & \\
\hline 30 & $\mathrm{Sx}$ & 6.15 & 259.465 & 259.4967 & $\ldots$ & $216 \pm 66.9$ & $\ldots$ & $\mathrm{b}$ \\
\hline 31 & S XI & 6.25 & 188.663 & 188.6753 & $\ldots$ & $56.4 \pm 14.0$ & $\ldots$ & \\
\hline 32 & S XI & 6.25 & 247.143 & 247.1594 & $\ldots$ & $151 \pm 53.3$ & $\ldots$ & $\mathrm{a}, \mathrm{c}$ \\
\hline 33 & S XI & 6.25 & 281.364 & 281.4021 & $\ldots$ & $208 \pm 79.8$ & $\ldots$ & $\mathrm{a}, \mathrm{c}$ \\
\hline 34 & S XI & 6.25 & 285.820 & $\begin{array}{l}285.5875 \\
285.8226\end{array}$ & $\ldots$ & $132 \pm 44.3$ & $\ldots$ & $\mathrm{a}$ \\
\hline 35 & S XII & 6.30 & 212.088 & 212.1205 & $\ldots$ & $78.6 \pm 16.7$ & $\ldots$ & \\
\hline 36 & S XII & 6.35 & 218.196 & 218.2005 & $\ldots$ & $156 \pm 29.9$ & $\ldots$ & $\mathrm{b}$ \\
\hline 37 & S XII & 6.35 & 288.415 & 288.4210 & $136 \pm 35.9$ & $203 \pm 54.7$ & $\ldots$ & \\
\hline 38 & S XII & 6.35 & 299.553 & 299.5407 & $\ldots$ & $\ldots$ & $34.7 \pm 11.5$ & \\
\hline 39 & S XIII & 6.40 & 256.666 & 256.6852 & $\ldots$ & $802 \pm 108$ & $\ldots$ & \\
\hline 40 & S XIV & 6.50 & 417.688 & 417.6611 & $67.1 \pm 12.8$ & $\ldots$ & $\ldots$ & \\
\hline 41 & Ar XI & 6.30 & 188.821 & 188.8060 & $\ldots$ & $28.3 \pm 10.7$ & $\ldots$ & a \\
\hline 42 & Ar XIV & 6.55 & 187.962 & 187.9690 & $\ldots$ & $26.3 \pm 8.60$ & $\ldots$ & \\
\hline 43 & Ar XIV & 6.55 & 194.401 & 194.3960 & $\ldots$ & $18.5 \pm 6.80$ & $\ldots$ & $\mathrm{a}$ \\
\hline 44 & Ca VII & 5.70 & 342.435 & $\begin{array}{l}342.3953 \\
342.8179\end{array}$ & $\ldots$ & $\ldots$ & $7.3 \pm 3.10$ & $\mathrm{a}$ \\
\hline 45 & Ca XIV & 6.45 & 186.616 & 186.6103 & $\ldots$ & $77.4 \pm 15.5$ & $\ldots$ & \\
\hline 46 & Ca XIV & 6.55 & 193.872 & 193.8661 & $\ldots$ & $76.2 \pm 10.7$ & $\ldots$ & \\
\hline 47 & $\mathrm{Caxv}$ & 6.65 & 200.978 & 200.9719 & $\ldots$ & $118 \pm 20.0$ & $\ldots$ & \\
\hline 48 & Ca XVII & 6.70 & 192.883 & 192.8532 & $\ldots$ & $39.0 \pm 10.7$ & $\ldots$ & b \\
\hline 49 & $\mathrm{Ni}$ XVI & 6.45 & 194.026 & 194.0460 & $\ldots$ & $19.6 \pm 3.50$ & $\ldots$ & \\
\hline 50 & $\mathrm{Ni}$ XVI & 6.45 & 239.504 & 239.5080 & $\ldots$ & $156 \pm 61.0$ & $\ldots$ & $\mathrm{a}$ \\
\hline 51 & Ni XVII & 6.50 & 249.168 & 249.1856 & $\cdots$ & $587 \pm 81.8$ & $\ldots$ & \\
\hline 52 & $\mathrm{Ni}$ XVIII & 6.55 & 291.983 & 291.9840 & $123 \pm 28.7$ & $481 \pm 68.7$ & $\ldots$ & \\
\hline 53 & Ni XVIII & 6.55 & 320.559 & 320.5660 & $46.6 \pm 6.59$ & $\ldots$ & $85.3 \pm 14.3$ & \\
\hline 54 & Cr XIII & 6.20 & 328.257 & 328.2680 & $57.7 \pm 7.32$ & $\ldots$ & $62.9 \pm 10.3$ & \\
\hline 55 & Cr XIV & 6.25 & 389.903 & 389.8640 & $55.9 \pm 12.3$ & $\ldots$ & $\ldots$ & \\
\hline 56 & $\mathrm{Mn} \mathrm{XV}$ & 6.35 & 361.007 & 361.0120 & $29.1 \pm 5.56$ & $\ldots$ & $\ldots$ & \\
\hline
\end{tabular}

Notes.

a These detections are $<3 \sigma ;{ }^{*}$ indicates only one data set value is problematic.

${ }^{\mathrm{b}}$ CHIANTI indicates that these lines are blended; they are not used in our analysis.

${ }^{\mathrm{c}}$ Predicted intensity is $>5 \sigma$ low compared with observed; * indicates only SERTS-95 value is problematic. 


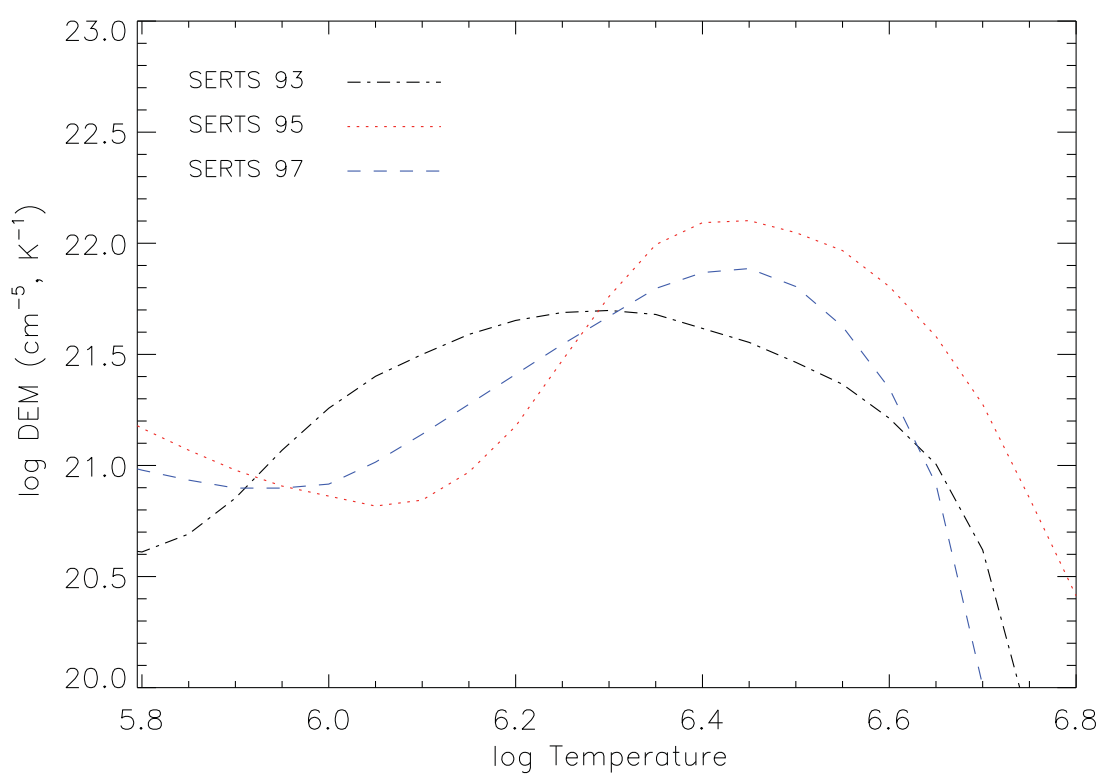

Figure 1. DEM distributions for the active regions observed during the three SERTS rocket flights constructed with the SERTS iron lines and constrained at the high-temperature end by the SXT broadband data which are dominated by iron lines at these temperatures. These distributions conform to the definition of DEM as $n_{e}^{2} d l / d T$ and use the ionization fractions of Bryans et al. (2009) and the recommended coronal abundances of Schmelz et al. (2012).

(A color version of this figure is available in the online journal.)

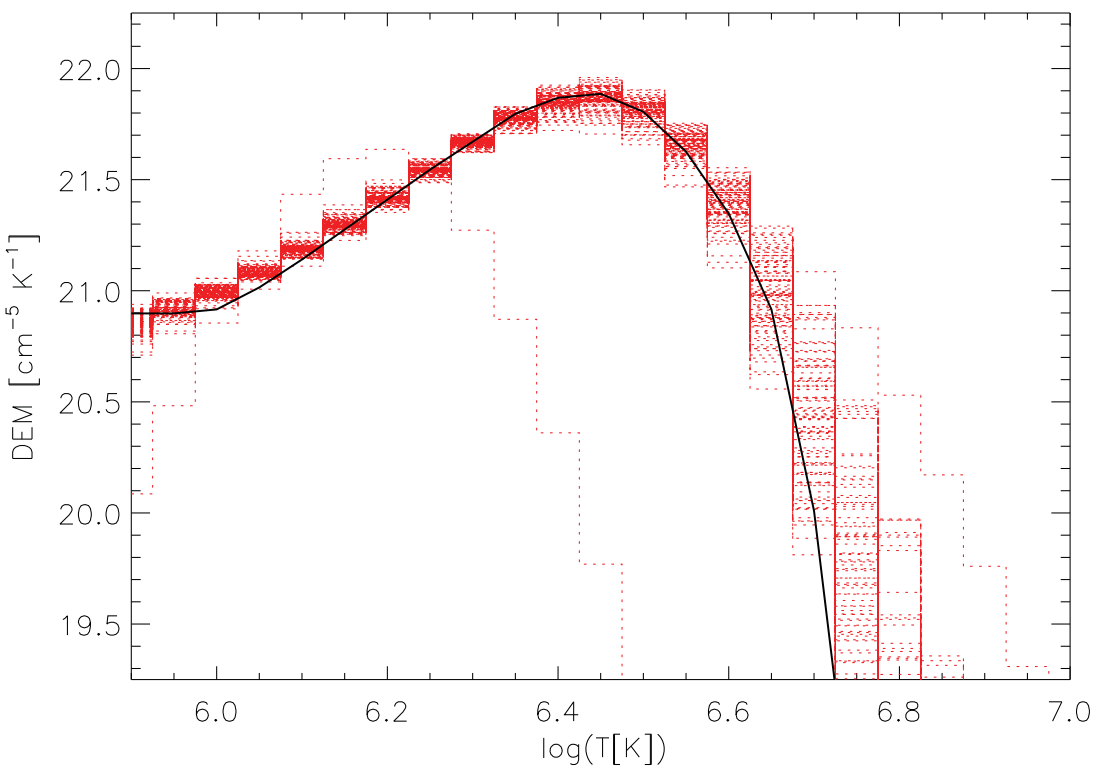

Figure 2. Comparison of the DEM_manual curve in black and the Monte Carlos from xrt_dem_iterative2 in red for the SERTS-97 data. The agreement between the different methods and the tightness of the Monte Carlos are encouraging, showing that the DEM shape is indeed determined by the data.

(A color version of this figure is available in the online journal.)

of Monte Carlos is tight, even at high temperatures, indicating that the DEM is now well constrained. In the following analysis, we use the DEM results obtained with the combination of the SERTS iron lines and the SXT data.

\subsection{Deriving the Electron Density from DEM Analysis}

The density is a key parameter of the coronal plasma, essential for trying to understand the fundamental physics of complex phenomena such as coronal heating and loop dynamics. Plasma densities are usually determined from the ratio of intensities of two spectral lines, ideally from the same element and ionization state to avoid having to know the elemental abundances and the ionization fractions. For example, Brosius et al. (1996) used this line-ratio method to calculate the electron density for the core of AR 7563 from several pairs of iron lines observed during SERTS-93. Their results showed a wide range of densities $\left(1.5 \times 10^{9}\right.$ to $\left.2.0 \times 10^{10} \mathrm{~cm}^{-3}\right)$, even within the same ion species (Fe XIII) where one might expect more consistency in the absence of systematic uncertainties in the atomic data.

The plots in Figure 4 represent the SERTS-93 data set only, but the results apply more generally to SERTS-95 and SERTS-97 as well. These panels show the predicted-to-observed intensity ratios for each of the iron lines listed in Table 1, for three different adopted values of the plasma electron density. The black stars represent spectral lines that are not sensitive to density changes (in this range), the blue squares represent lines whose predicted-to-observed intensity ratio decreases with 

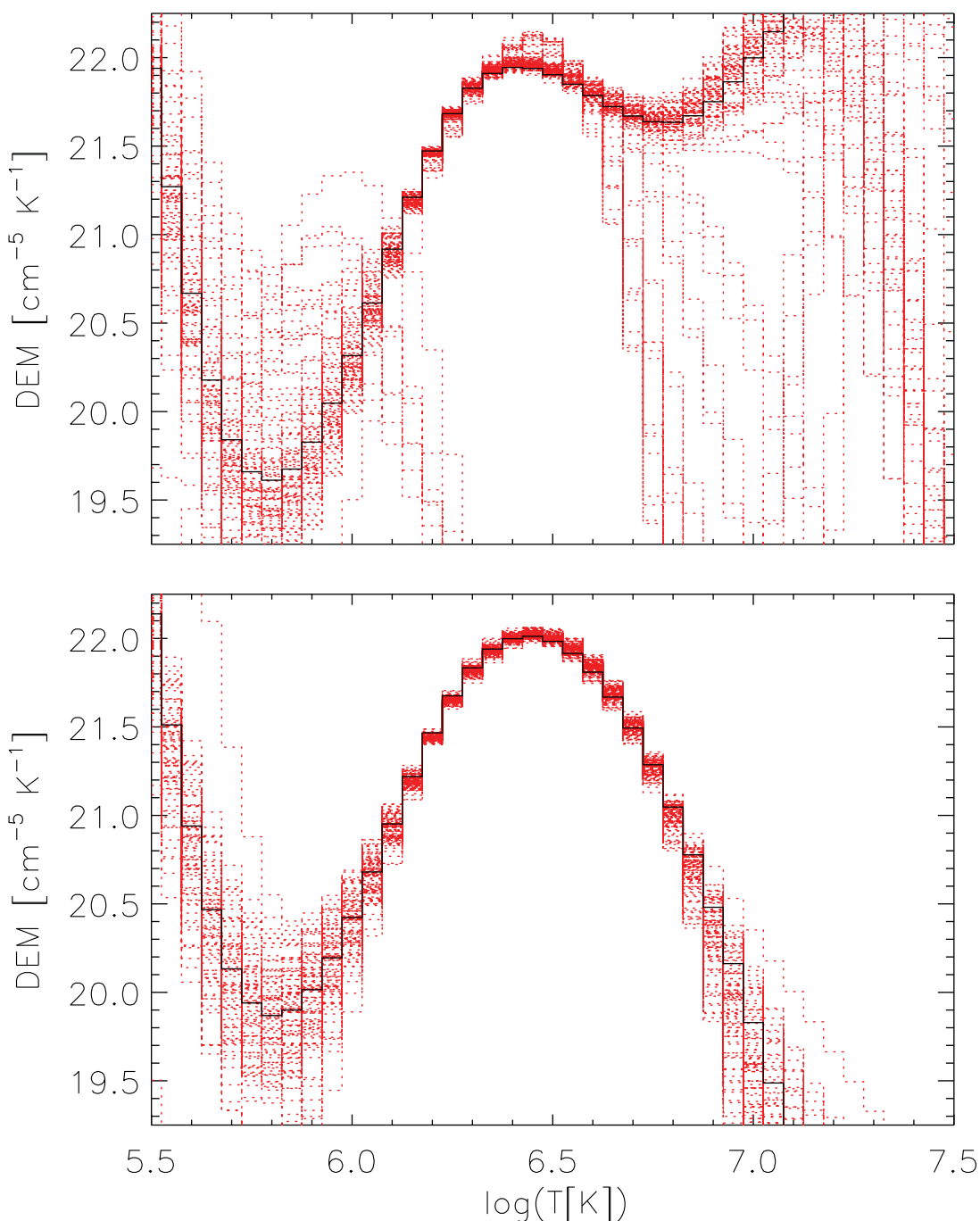

Figure 3. DEM results for the SERTS-95 data using xrt_dem_iterative 2 with the SERTS iron lines only (top panel) and the SERTS iron lines plus SXT data (bottom panel). The minimum $\chi^{2}$ DEM is shown in black and the Monte Carlos are in red. The Monte Carlos begin to deviate above $\log T=6.6$ in the top panel but are tight in the bottom panel, which illustrates the need for the SXT high-temperature constraint (see the text).

(A color version of this figure is available in the online journal.)

increasing density, and red diamonds represent lines whose predicted-to-observed intensity ratio increases with increasing density. In the top plot $\left(n_{e}=2 \times 10^{8} \mathrm{~cm}^{-3} ; \bar{\chi}^{2}=15.9\right)$, the squares are too high and the diamonds are too low; the reverse is true for the bottom plot $\left(n_{e}=2 \times 10^{10} \mathrm{~cm}^{-3} ; \bar{\chi}^{2}=11.1\right)$. The middle plot $\left(n_{e}=2 \times 10^{9} \mathrm{~cm}^{-3} ; \bar{\chi}^{2}=2.7\right)$ gives the best match of predicted-to-observed intensities for the three densities tested. The best-fit densities using this method are $n_{e}=2.2 \times 10^{9} \mathrm{~cm}^{-3}$ with $\bar{\chi}^{2}=2.3$ for SERTS-93, $n_{e}=2.9 \times 10^{9} \mathrm{~cm}^{-3}$ with $\bar{\chi}^{2}=$ 7.4 for SERTS-95, and $n_{e}=2.3 \times 10^{9} \mathrm{~cm}^{-3}$ with $\bar{\chi}^{2}=1.5$ for SERTS-97.

Using these densities, the total column emission measure calculated in the previous subsection, and assuming a path length of (1) $\sim 2 \times 10^{9} \mathrm{~cm}$, a typical loop width, or (2) $\sim 2 \times 10^{10} \mathrm{~cm}$, a typical loop height (Bray et al. 1991), we can calculate the geometric density, $n_{g}$, and the resulting filling factor for each SERTS observation: (1) $n_{g}=2.4 \times 10^{9} \mathrm{~cm}^{-3}$ and $\mathrm{ff}=1.1$ for SERTS-93, $n_{g}=3.7 \times 10^{9} \mathrm{~cm}^{-3}$ and $\mathrm{ff}=1.3$ for SERTS-95, and $n_{g}=2.8 \times 10^{9} \mathrm{~cm}^{-3}$ and $\mathrm{ff}=1.2$ for SERTS-97. (2) $n_{g}=7.8 \times 10^{8} \mathrm{~cm}^{-3}$ and $\mathrm{ff}=0.35$ for SERTS-93, $n_{g}=1.2 \times 10^{9} \mathrm{~cm}^{-3}$ and $\mathrm{ff}=0.41$ for SERTS-95, and $n_{g}=8.7 \times 10^{9} \mathrm{~cm}^{-3}$ and $\mathrm{ff}=0.38$ for SERTS-97.

This illustrates the problem with the geometric method: it depends strongly on the estimate of the path length, a parameter which is not easy to pin down. For typical values, the filling factor can range from $\sim 30 \%$ to $40 \%$ up to one, within the uncertainties. These values are consistent with the range of filling factors for active regions present in the literature.

Our approach for constraining the density seemed promising for SERTS-93 (Figure 4) and SERTS-97, but the agreement between the observed and predicted values for some iron lines in the SERTS-95 data set was so bad that we would never get close to an acceptable value for $\bar{\chi}^{2}$ if we used all the available lines. A number of line predictions might well be inaccurate because of problems with the atomic data or ionization fraction and there might be systematic errors for observed intensities with poor signal to noise. Therefore, we investigated the following possibility: could we get an acceptable fit for a single density by successive elimination of lines with the greatest predictedto-observed discrepancies from the derived DEM? If we could 

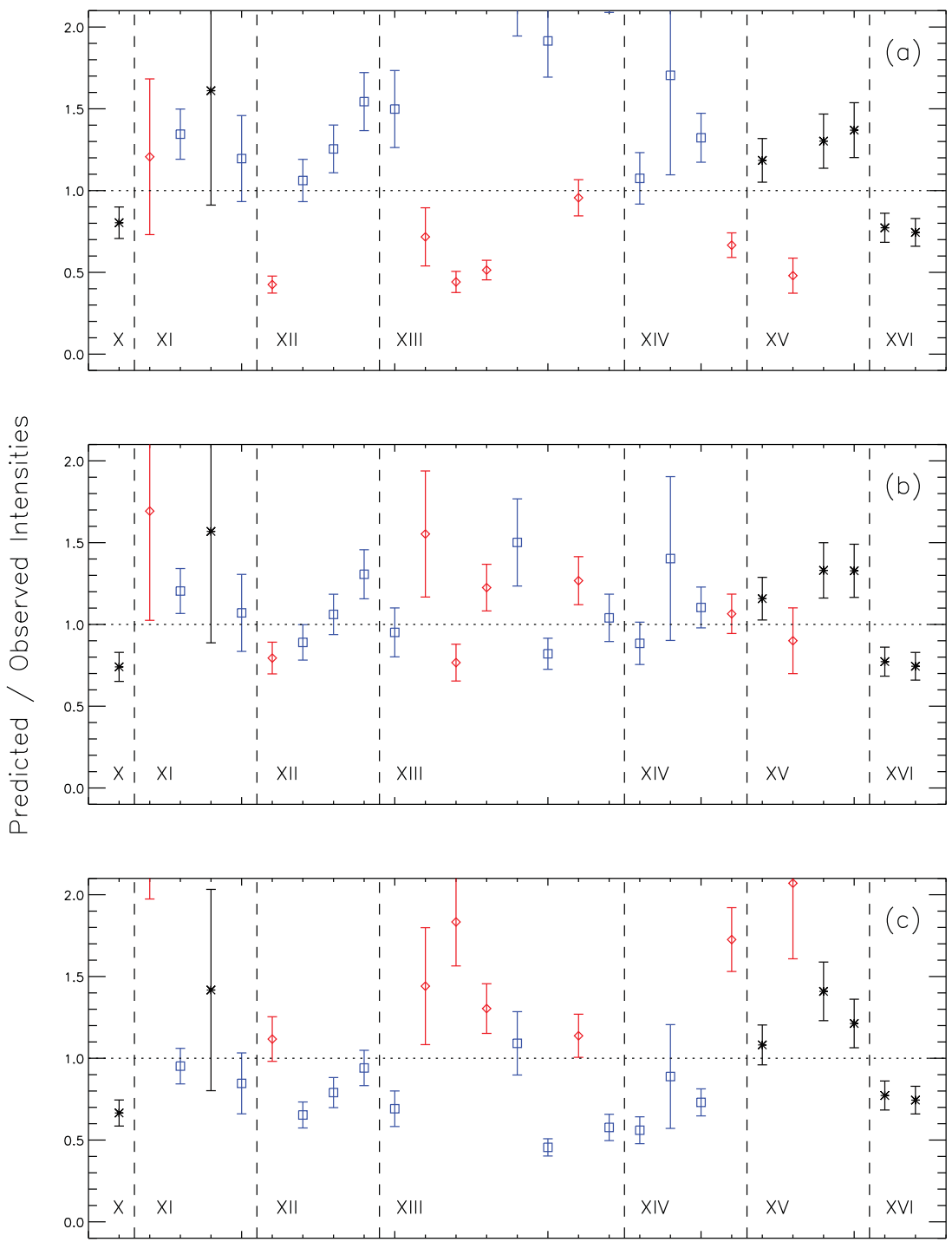

Figure 4. Predicted-to-observed intensity ratios for the SERTS-93 iron lines listed in Table 1. The points are plotted in temperature order for different values of the electron density: (a) $n_{e}=2 \times 10^{8} \mathrm{~cm}^{-3}$ and $\bar{\chi}^{2}=15.9$, (b) $n_{e}=2 \times 10^{9} \mathrm{~cm}^{-3}$ and $\bar{\chi}^{2}=2.7$, and (c) $n_{e}=2 \times 10^{10} \mathrm{~cm}^{-3}$ and $\bar{\chi}^{2}=11.8$. The black stars represent spectral lines that are not sensitive to density changes (in this range), the blue squares represent lines whose predicted-to-observed intensity ratio goes down as density increases, and red diamonds represent lines whose predicted-to-observed intensity ratio goes up as density increases.

(A color version of this figure is available in the online journal.)

achieve $\bar{\chi}^{2} \simeq 1$ while keeping adequate temperature coverage with a sufficient number of density-sensitive lines, that would allow us to estimate $1 \sigma$ uncertainties on the density by finding the density values for which the total $\chi^{2}$ increased by unity from the best-fit value (i.e., $\Delta \chi^{2}=\chi_{\min }^{2}+1$; see, e.g., Bevington $\&$ Robinson 1992, pp 144-147).

The results of this approach for the SERTS-95 data are shown in Figure 5. The dotted curve shows the initial $\bar{\chi}^{2}$ distribution where we used all available iron lines, the dot-dashed curve shows the changes if we remove lines for which the predictedto-observed intensity ratio was $>5 \sigma$ from unity, the dashed curve shows the changes if we remove lines for which the predicted-to-observed intensity ratio was $>3 \sigma$ from unity, and the solid curve shows the final distribution where we remove even those lines with predicted-to-observed intensity ratios that were $>2 \sigma$ from unity. This method left adequate coverage for fitting density over the temperature range $6.0 \leqslant \log T \leqslant 6.4$. The best-fit density is given by the lowest point in the solid curve. The density for the SERTS-95 data set obtained using this method is $n_{e}=\left(6.9_{-5.2}^{+26}\right) \times 10^{9} \mathrm{~cm}^{-3}$. As noted above, the quoted uncertainties are $1 \sigma$ and correspond to an increase of one in $\Delta \chi^{2}$.

There is no a priori reason to think that all the EUV/X-ray emission from the core of a given active region would come from loops with a single mean electron density across a broad temperature regime. Rather, these DEM-weighted values are exactly those densities responsible for the bulk of the observed emission. Because the method uses a large number of spectral lines simultaneously, it is not heavily biased by potential atomic data uncertainties in any given line ratio. We suggest that determining the DEM-weighted density might be a powerful density diagnostic tool, complementary to the standard lineratio method. In addition, DEM forward-fitting may provide a straightforward method to estimate realistic uncertainties.

\subsection{Problematic Lines and Uncertainties}

Figure 6 shows the predicted-to-observed intensity ratios for the SERTS-93 lines. The top panel shows the iron lines, the middle panel shows the non-iron lines with Schmelz et al. (2012) abundances, and the bottom panel shows the non-iron lines with 


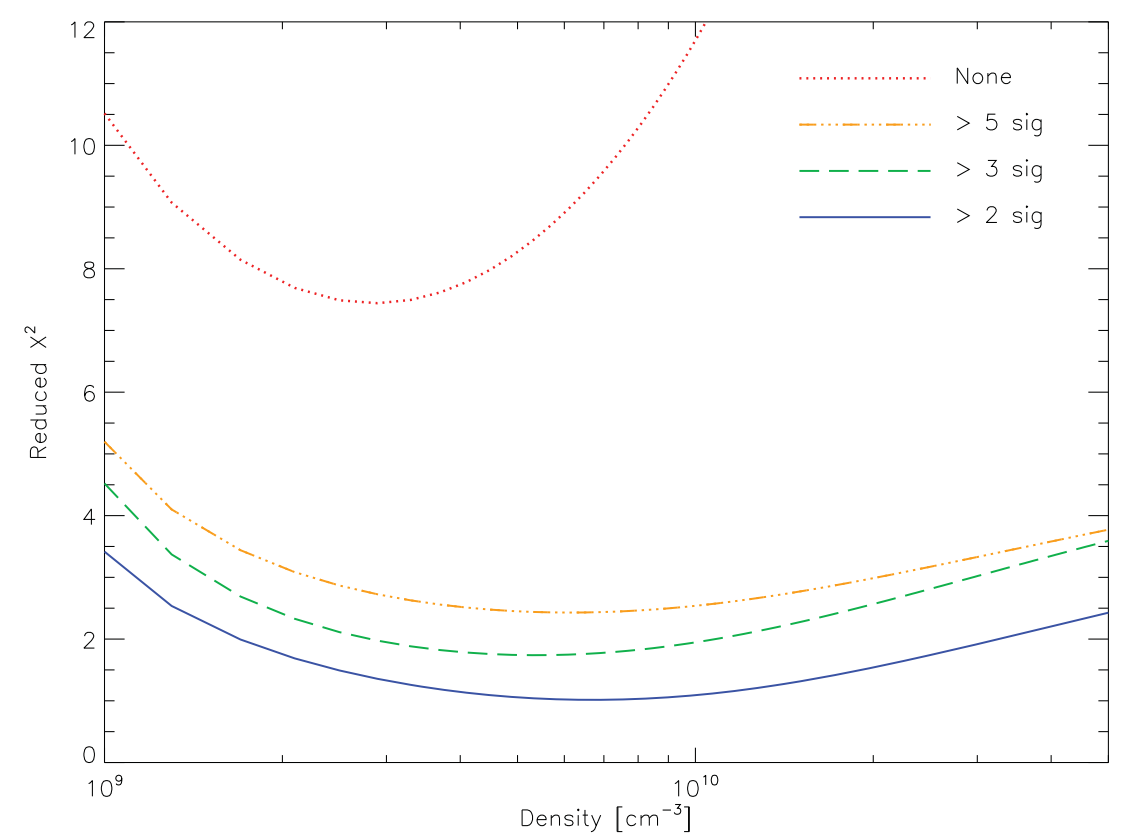

Figure 5. Plots of $\bar{\chi}^{2}$ as a function of electron density for SERTS-95. The dotted curve shows the initial $\bar{\chi}^{2}$ distribution, the dot-dashed curve shows the changes if we remove lines where the predicted-to-observed intensity ratio was $>5 \sigma$ from unity, the dashed curve shows the changes if we remove lines where the predicted-toobserved intensity ratio was $>3 \sigma$ from unity, and the solid curve shows the final distribution where we remove lines where the predicted-to-observed intensity ratio was $>2 \sigma$ from unity. The minimum in the solid curve represents the best-fit density, where the $\bar{\chi}^{2}$ has dropped to $\sim 1$.

(A color version of this figure is available in the online journal.)

the abundances of the various elements adjusted to minimize the $\bar{\chi}^{2}$. The peak formation temperatures of the lines generally increase from left to right across the plot. Figures 7 and 8 show similar results for the SERTS-95 and SERTS-97 lines.

There are many SERTS detections that are $<3 \sigma$ in significance. These are labeled with a " 1 " in the last column of Tables 1 and 2. Lines that are blended are labeled with a " 2 " and are not used in the DEM analysis. Lines where the predicted-toobserved intensity ratios are $>5 \sigma$ lower than unity are labeled with a " 3 " and lines where the predicted-to-observed intensity ratios are $>5 \sigma$ higher than unity are labeled with a "4." Adjusting the DEM curve, the electron density, or the elemental abundance did not improve the fit. The problems with these lines may result from unidentified blends or poor signal to noise. There are also two Fe XVII lines where the differences between the observed and predicted values for the intensity are so large that we suspect that these lines may be misidentified, blended, or require improved atomic data. These lines were also excluded from the DEM analysis.

The notes column of Tables 1 and 2 shows that there is only one line labeled with a " 4 ," the Fe XIV line at 219.121 A observed as part of SERTS-95. It is a relatively strong, well-detected line and appears to be properly identified. A high predicted-toobserved intensity ratio could result from resonance scattering, but this seems unlikely (see, e.g., Brickhouse \& Schmelz 2006). A more plausible explanation may be related to the density sensitivity since it does not appear to agree with the results of the other SERTS-95 Fe XIV lines. Many more lines, however, are labeled with a " 3 " in the notes column indicating that the predicted-to-observed intensity ratios are too low. This implies that the problem may be either missing lines (unknown blends) or missing flux from processes that are not yet accounted for. This problem is more prevalent for the SERTS-95 lines, perhaps because this wavelength range is not as well studied.

\subsection{Relative Elemental Abundances}

We were interested in seeing how well the three DEM curves in Figure 1, created with iron lines alone, predicted the intensities for the non-iron lines. Initially, the unadjusted abundance values (relative to the iron abundance) from Schmelz et al. (2012) were used for each separate SERTS data set. The results of the analysis are shown in the three middle panels of Figures 6-8. We discuss each element separately below.

Magnesium. The DEM curves optimized for the iron lines do a good job of reproducing the magnesium line intensities. There is one caveat, however. Many of the lines considered here are from the $\mathrm{Mg} \mathrm{V}-\mathrm{Mg}$ VIII ionization stages, with peak formation temperatures of $\log T=5.5-5.9$. We have to bootstrap the iron-only DEM curves to slightly lower temperatures. As a result, there is some uncertainty in the exact placement of the low-temperature end of the DEM curve, and therefore, the exact value of the magnesium abundance required to bring the observed and predicted values for the intensities into agreement. There is, however, Fe X-Mg IX overlap for SERTS-93, each with a peak formation temperature of $\log T=6.0$, as well as Fe VIII and Fe IX in SERTS-95. In summary, the SERTS data give us no reason to think that the recommended abundance value for magnesium from Schmelz et al. (2012) needs to be adjusted.

Aluminum. The $\mathrm{Al} x$ line at $332.784 \AA$ is the only aluminum line observed, but it is listed in all three data sets. There are two strong detections: $8 \sigma$ in SERTS-93 and $6.2 \sigma$ in SERTS-97. The SERTS-95 detection is only $4 \sigma$. The line has a peak formation temperature of $\log T=6.10$, so its predicted intensity is well constrained by the iron-line DEMs. The SERTS-93 aluminum abundance needs to be reduced to $64 \% \pm 10 \%$ of the Schmelz et al. (2012) recommended value in order to minimize $\chi^{2}$, whereas the SERTS-95 value must be increased to $190 \% \pm$ $70 \%$. The 1997 value is consistent with the recommended 

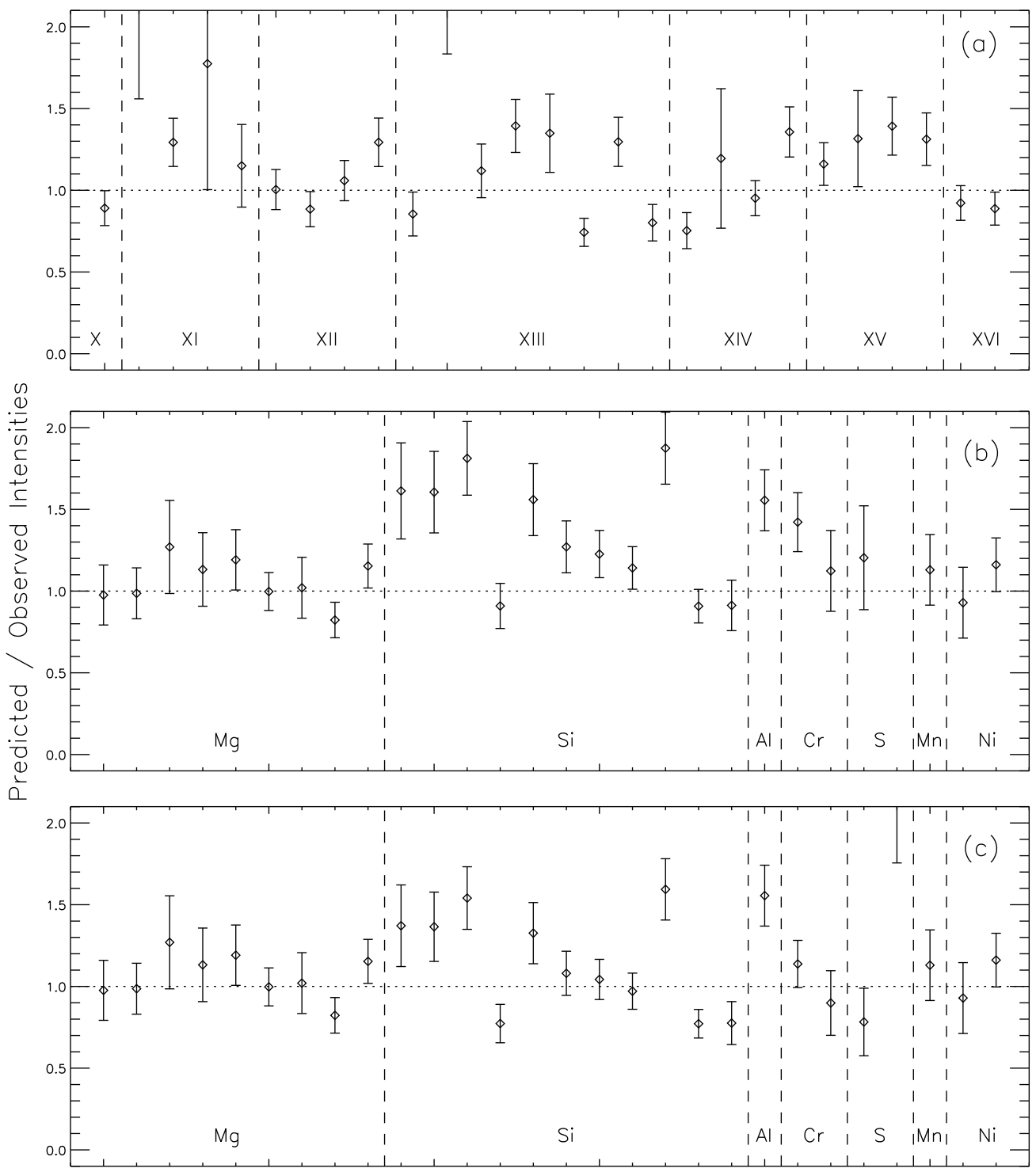

Figure 6. Predicted-to-observed intensity ratios for the spectral lines observed by SERTS-93. Top: the iron lines only; middle: the non-iron lines with the abundance normalization of Schmelz et al. (2012); and bottom: the non-iron lines with the abundance values adjusted to best match the total SERTS data set.

abundance. Strictly speaking, the SERTS results themselves are not mutually consistent. However, we hesitate to draw strong conclusions based on a single line, and do not necessarily consider this difference to be the result of abundance variability.

Silicon. There were more lines of silicon than of any other element except iron, with 11, four, and nine usable lines in the SERTS-93, -95, and -97 data sets, respectively. These silicon lines were produced from a variety of ionization stages from Si VIII to Si XI, with a range of peak formation temperatures of $\log T=5.9-6.2$. The iron-only DEM curves do a good job of constraining all of these except for the SERTS-93 and -97 Si VIII, but these were both bootstrapped using cooler magnesium lines (see above). The SERTS-93 and -97 data indicate that the silicon abundance should be lowered to $85 \% \pm 10 \%$ and $75 \% \pm 10 \%$ of the Schmelz et al. (2012) recommended value, respectively. The four SERTS-95 silicon lines do not agree with the scaling or with each other. We suspect that the SERTS-95 Si VIII $214.76 \AA$ line and the Si x $261.04 \AA$ line may be blended with lines that are not yet part of the CHIANTI database. If we eliminate these lines from our analysis, our results indicate that the silicon recommended abundance should be lowered to $\sim 85 \%$ of its current value.

Sulfur. The elemental abundance of sulfur is notoriously difficult to pin down because it (along with phosphorus) is the so-called intermediate-FIP element. Two SERTS-95 S XI lines at 247.14 and $281.36 \AA$ are consistent with unidentified blends and inconsistent with the results of the other sulfur SERTS lines. If we eliminate these lines from our analysis, our results indicate that the recommended sulfur abundance of Schmelz et al. (2012) is too high, and should be lowered to $\sim 65 \%$ of its current value.

Argon. Argon is the only high-FIP element in the data set. The only detections were from SERTS-95, and two of these were eliminated because they were weak ( $\leqslant 3 \sigma$ detection). The best-fit abundance $(102 \pm 33)$ for the single remaining line is consistent with the recommended value of Schmelz et al. (2012) but does not tightly constrain it.

Calcium. There is one weak $(<3 \sigma)$ Ca VII line in the SERTS97 data set and four lines formed at higher temperatures in the SERTS-95 data set. The Ca XIV line is not in the CHIANTI database and the CaXVII is blended. The remaining CaXIV 


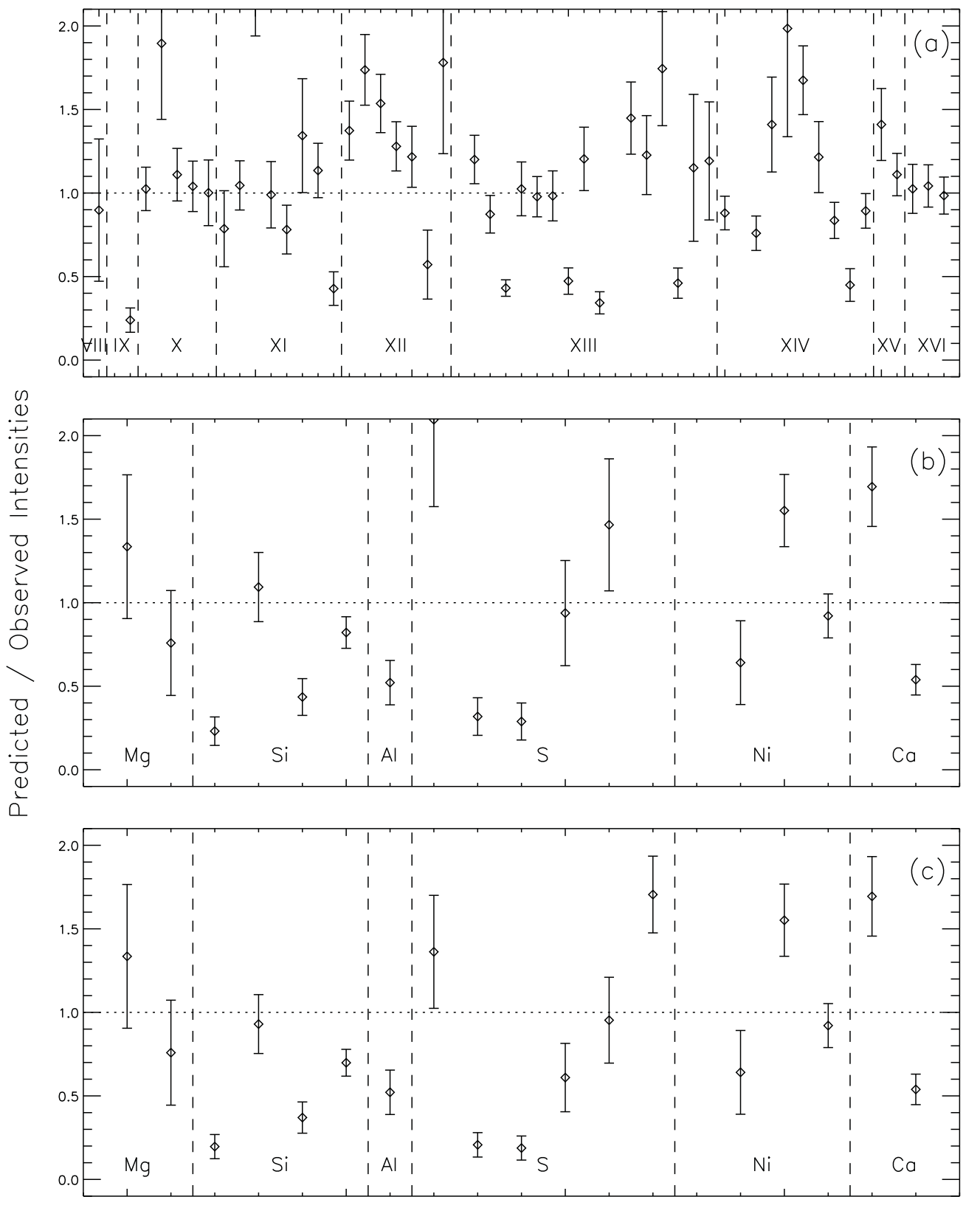

Figure 7. Same as Figure 6, but for SERTS-95.

and $\mathrm{Xv}$ lines are $5 \sigma$ detections, but Figure 7 shows that the predicted-to-observed intensity ratio for the first is high and the second is low. The available SERTS calcium data do not appear to present a viable case for changing the recommended value of Schmelz et al. (2012).

Nickel. The nickel lines from SERTS-93 and SERTS-95 are consistent with the Schmelz et al. (2012) recommended abundance value, with best-fit results of $106 \% \pm 10 \%$ and $87 \% \pm 16 \%$, respectively.

Chromium. There are two chromium lines in the SERTS-93 data set and one in the SERTS-97 data set. All are consistent with the Schmelz et al. (2012) recommended value.

Manganese. There is one Mn Xv line in the SERTS-93 data set. Schmelz et al. (2012) had no results for manganese itself, but if we apply their algorithm for low-FIP elements to manganese, we find that the resulting abundance value is consistent with the SERTS data (see Figure 6).

\section{DISCUSSION}

\subsection{Comparison of Forward-fitting with Inverse DEM Curves}

The differences between our SERTS-93 DEM curve in Figure 1 and the distribution published originally by Brosius et al. (1996, see their Figure 8(a)) were discussed in detail by Schmelz et al. (1999) and are summarized here. Both analyses used the same SERTS data, but the normalizations and the shapes of the two resulting curves are distinctly different. In our analysis, the normalization of the curve conforms to the definition of DEM as $n_{e}^{2} d l / d T$; in addition, we have used the recommended coronal abundances of Schmelz et al. (2012) instead of the old values of Feldman (1992). The main difference in the shapes of the two curves can be attributed to our use of cotemporal data from three SXT broadband filters (thin Al, $\mathrm{AlMgMn}$, and thick $\mathrm{Al}$ ) as a high-temperature constraint (see Schmelz et al. 1999). The Fe XV and Fe XVI SERTS lines as well 


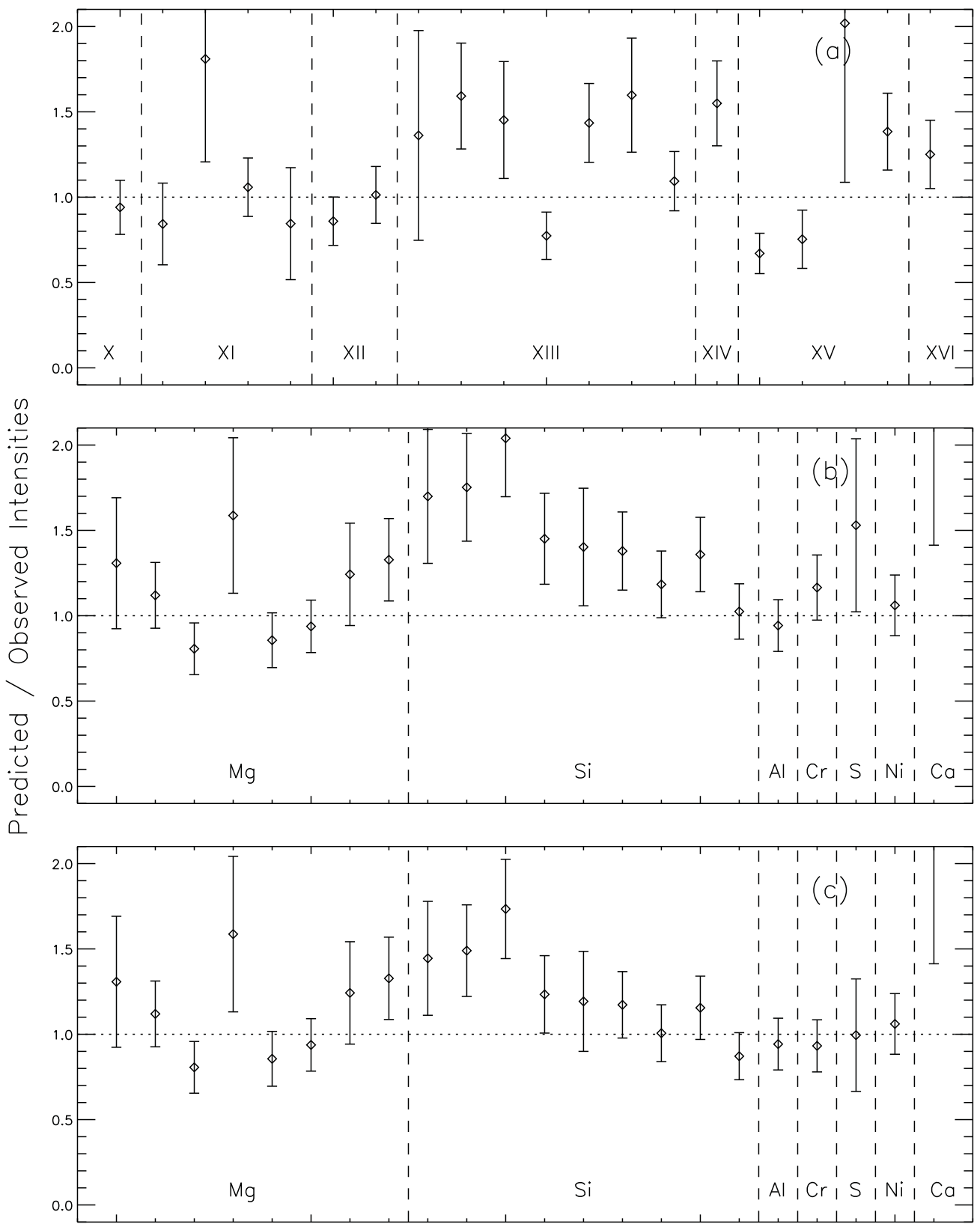

Figure 8. Same as Figure 6, but for SERTS-97.

as the Ni XVIII lines were in agreement with the SXT data. The predicted-to-observed ratios of the two weak Fe XVII lines at 347.8 and $350.5 \AA$, however, were still too low by more than a factor of two and would have required an unrealistically large cross-calibration correction factor to bring them into agreement. Since this discrepancy could not be attributed to statistical or systematic uncertainties, Schmelz et al. (1999) suggested that the atomic physics for these lines be reexamined.

There is no DEM curve for the SERTS-95 data published previously by the SERTS team or-to the best of our knowledge-by anyone else, to compare with our DEM curve. Our curve, shown by the dashed line in Figure 1, is well covered from $\log T=5.9-6.4$, by multiple lines from each ionization stage of iron from Fe IX through Fe XVI inclusive. There are also two Fe viII lines, but one is blended and one is a $<3 \sigma$ detection, so extending the DEM curve to lower temperatures is problematic. As in the SERTS-93 case, plasma at higher temperatures is nicely constrained by the SXT broadband filter data (see Figure 3).

For the SERTS-97 data, after we adjusted the normalization of the DEM curve obtained by Brosius et al. (2000) as discussed above for the SERTS-93 data, our respective distributions are essentially identical for temperatures above $\log T=5.8$ (cf. their Figure 4 and the dash-dotted line in our Figure 1). We attribute this agreement to the fact that Brosius et al. chose not to use the discrepant Fe XVII lines at 347.8 and $350.5 \AA$ mentioned above in their SERTS-97 DEM analysis. This agreement is encouraging since their method uses an automated inversion and ours uses two different forward-fitting approaches, and there have not been many direct comparisons of DEM curves from the two methods for real data published in the literature.

Figure 1 shows that different active regions have different DEM shapes (in addition, see Warren et al. 2012; Schmelz $\&$ Pathak 2012). The agreement between the results from 
DEM_manual and xrt_dem_iterative2 as well as the older inversion results (where available) described above gives us confidence that the SERTS plus SXT data together provide a robust determination of the curve, and that the differences are not attributable to possible idiosyncrasies in the DEM methods. Some of these differences are most likely related to the core heating of the region, whether it is steady (Winebarger et al. 2011) or nanoflare (Tripathi et al. 2011) heating, for example. With these new DEM techniques and the improved atomic data available in CHIANTI v.7, we expect to make significant progress in obtaining the more accurate physical quantities needed to understand such important physical phenomena as active region heating.

\subsection{Absolute Elemental Abundances}

From a survey of absolute coronal abundance values published in the literature, Schmelz et al. (2012) found that the abundances of elements with low FIP were enhanced over the photospheric values reported by Caffau et al. (2011) and Lodders et al. (2009) by a mean value of $2.14 \pm 0.46$ (rather than the factor of four proposed by Feldman 1992); and the abundances of high-FIP elements were depleted by a mean factor of $0.71 \pm 0.14$ (rather than 0.25 as proposed by Meyer 1985). The resulting set of recommended coronal abundances of Schmelz et al. (2012) is approximately the geometric mean of the Feldman (1992) and the Meyer (1985) abundance sets.

Absolute abundances in the corona are still a subject of some controversy. For example, the measured differences between Raymond et al. (1997) and Feldman et al. (1998) for streamer abundances may be the result of gravitational settling. The different iron abundance values obtained by White et al. (2000) using Solar and Heliospheric Observatory CDS iron lines and thermal bremsstrahlung emission from the hydrogen continuum measured by the Very Large Array (VLA) are a factor of two higher than those of Zhang et al. (2001) who did a similar analysis using EIT and the VLA. These results relied heavily on the instrument cross-calibrations, but there is no way to tell which was right (or even which was better.) The one thing we seem to know for certain is that coronal abundances differ from their photospheric counterparts in a way that depends primarily on FIP, although under certain conditions, additional physical mechanisms may need to be taken into account (see, e.g., Schmelz 1993).

There is also evidence for abundance variability in flares (e.g., Sylwester et al. 1984), from active region to active region (e.g., Strong et al. 1988), and between and within different kinds of coronal structures (e.g., Widing \& Feldman 1989). Some of the apparent variations reported in these older papers might be attributable to instrument calibration problems, atomic physics uncertainties, or temperature effects. A deeper understanding of these possible variations might result from our improved understanding of the thermal structure of the corona, better multithermal analysis techniques (e.g., DEM_manual and xrt_dem_iterative2, with high- and low-temperature constraints as appropriate), and most importantly, updated atomic modeling for coronal emission lines now available in CHIANTI v.7. In fact, we tried to do this very analysis of SERTS plus SXT data about 10 years ago with atomic data from CHIANTI v. 2 and the cruder DEM techniques we had available at the time. The results were sufficiently disappointing that we put the project on hold until about six months ago.

Although it is impossible to provide a single model satisfying all observations, and although it is vital to account for the possibility of abundance variability, it is often useful to begin a new research project (like this one) with a given set of abundances. The results presented here support use of the recommended coronal abundances of Schmelz et al. (2012), which were derived from work by several groups using various types of data from different instruments on numerous spacecraft, as the default values in future investigations.

\subsection{FIP Effect}

The SERTS results reported herein suggest that two small changes might need to be made to the Schmelz et al. (2012) coronal abundance values. Silicon could be lowered to $\sim 85 \%$ of its current value, which is within the $1 \sigma$ uncertainty. Sulfur, on the other hand, might need to be lowered to $\sim 65 \%$ of its current value. The lower sulfur abundance is consistent with the spectroscopic measurements from Veck \& Parkinson (1981) and Fludra \& Schmelz (1999), but at odds with the SEP data from Reames (1995) and the solar wind data from von Steiger et al. (2000, 2010). Abundance results for this intermediate-FIP element are difficult to pin down, and further analysis will be required to sort out this problem.

One thing the SERTS data do not provide, however, is strong observational evidence of the traditional FIP step, i.e., that the coronal-to-photospheric abundance ratios of low-FIP elements seem to be enhanced relative to those of high-FIP elements. This is because the only high-FIP coronal detections in the data sets were of the argon lines from SERTS-95, which were simply too weak to provide strong constraints on the coronal abundance. The other SERTS high-FIP detections were for transition region lines of neon and oxygen. These lines were too cool to be included in our DEM analysis, which was constrained by the SERTS iron lines (Table 1) and the SXT data. Strong coronal lines from high-FIP elements tend to have higher energies than the SERTS lines, so future joint EUV-Xray spectroscopy observations would be required to address this issue satisfactorily.

\section{CONCLUSIONS}

We have used the set of recommended coronal abundances from Schmelz et al. (2012) for our multithermal analysis of active region data. We feel that these abundances provide the best default characterization of the low corona when specific information on the composition of the coronal plasma under study is lacking. It gives the mean values of a large number of spectroscopic, particle, and solar wind observations from many different sources, and it typically reduces the uncertainties by a factor of two from either extreme of the two standard coronal abundance sets (Meyer 1985; Feldman 1992). The absolute normalization of the abundances is critical for determining the amount of emitting plasma, the radiative losses, and all of the physics depending on those quantities (such as the heating requirements and the energy budget).

We have used two iterative forward-fitting techniques to determine the DEM for the core structures in three active regions, using high-resolution EUV spectra from three SERTS rocket flights, in conjunction with high-temperature constraints from cotemporal broadband soft X-ray data from Yohkoh SXT. Elemental abundance uncertainties were not a major factor in deriving the shape of the distribution because we chose to work only with iron lines in the SERTS data, and iron lines dominate the SXT filter responses at active region temperatures. 
For the radiometrically calibrated SERTS-97 spectrum, the DEM curve we obtained with forward fitting is virtually identical to the curve derived from an inversion method by Brosius et al. (2000), for temperatures above $\log T=5.8$. This agreement is encouraging, especially since there have been few direct comparisons of DEM curves obtained from the two methods for real data published in the literature.

For each set of active region spectra, we were able to establish a characteristic electron density of the emitting plasma in the temperature range $6.0 \leqslant \log T \leqslant 6.4$ from the DEM-weighted density technique. We considered electron densities ranging from $10^{8}$ to $10^{10} \mathrm{~cm}^{-3}$ and found that the lowest $\bar{\chi}^{2}$ values were achieved with $n_{e}=2.2 \times 10^{9} \mathrm{~cm}^{-3}$ with $\bar{\chi}^{2}=2.3$ for SERTS-93, $n_{e}=2.9 \times 10^{9} \mathrm{~cm}^{-3}$ with $\bar{\chi}^{2}=7.4$ for SERTS-95, and $n_{e}=2.3 \times 10^{9} \mathrm{~cm}^{-3}$ with $\bar{\chi}^{2}=1.5$ for SERTS-97. In each case, an electron density significantly higher or lower than this best value made it increasingly difficult to derive an emission measure distribution with good agreement between theoretical and observed line intensities. We went one step further for SERTS-95, which had a number of bad results. We systematically eliminated these lines until we could get $\bar{\chi}^{2} \sim 1$, giving $n_{e}=\left(6.9_{-5.2}^{+26}\right) \times 10^{9} \mathrm{~cm}^{-3}$.

CHIANTI is a collaborative project involving the NRL (USA), the Universities of Florence (Italy) and Cambridge (UK), and George Mason University (USA). Solar physics research at the University of Memphis is supported by a Hinode subcontract from NASA/SAO as well as NSF ATM-0402729. The impetus for this work was a pilot study funded by a small Yohkoh Guest Investigator grant.

\section{REFERENCES}

Arnaud, M., \& Raymond, J. 1992, ApJ, 398, 394

Arnaud, M., \& Rothenflug, R. 1985, ApJS, 60, 425

Bevington, P. R., \& Robinson, D. K. 1992, Data Reduction and Error Analysis for the Physical Sciences (New York: McGraw-Hill)

Bray, R. J., Cram, L. E., Currant, C. J., \& Loughhead, R. E. 1991, Plasma Loops in the Solar Corona (Cambridge: Cambridge Univ. Press)

Brickhouse, N. S., \& Schmelz, J. T. 2006, ApJ, 636, 53

Brosius, J. W., Davila, J. M., \& Thomas, R. J. 1998, ApJS, 119, 255

Brosius, J. W., Davila, J. M., Thomas, R. J., \& Fossi, B. C. 1996, ApJS, 106,143
Brosius, J. W., Thomas, R. J., Davila, J. M., \& Landi, E. 2000, ApJ, 543, 1016 Bryans, P., Landi, E., \& Savin, D. W. 2009, ApJ, 691, 1540

Caffau, E., Ludwig, H.-G., Steffen, M., Freytag, B., \& Bonofacio, P. 2011, Sol. Phys., 268, 255

Dere, K. P., Landi, E., Mason, H. E., Fossi, B. C., \& Young, P. R. 1997, A\&AS, 125,149

Feldman, U. 1992, Phys. Scr., 46, 202

Feldman, U., Doschek, G. A., Laming, J. M., et al. 1998, ApJS, 504, 573

Fludra, A., \& Schmelz, J. T. 1999, A\&A, 348, 286

Landi, E., Del Zanna, G., Young, P. R., Dere, K. P., \& Mason, H. E. 2012, ApJ, 744, 99

Lodders, K., Palme, H., \& Gail, H. P. 2009, in Landolt-Börnstein, New Series, Vol. VI/4B, ed. J. E. Trümper (Berlin: Springer), 560, chap. 4.4

Meyer, J.-P. 1985, ApJS, 57, 173

Neupert, W. M., Epstein, G. L., Thomas, R. J., \& Thompson, W. T. 1992, Sol. Phys., 137, 87

Raymond, J. C., Kohl, J. L., Noci, G., Antonucci, E., et al. 1997, Sol. Phys., 175,645

Reames, D. V. 1995, Adv. Space Res., 15, 41

Schmelz, J. T. 1993, ApJ, 408, 373

Schmelz, J. T., Jenkins, B. S., Worley, B. T., et al. 2011a, ApJ, 731, 49

Schmelz, J. T., \& Pathak, S. 2012, ApJ, 756, 126

Schmelz, J. T., Reames, D. V., von Steiger, R., \& Basu, S. 2012, ApJ, 755, 33

Schmelz, J. T., Rightmire, L. A., Saar, S. H., Kimble, J. A., \& Worley, B. T. 2011b, ApJ, 738, 146

Schmelz, J. T., Saar, S. H., DeLuca, E. E., et al. 2009, ApJ, 693, L131

Schmelz, J. T., Saar, S. H., Nasraoui, K., et al. 2010, ApJ, 723, 1180

Schmelz, J. T., Saba, J. L. R., Strong, K. T., Wihter, H. D., \& Brosius, J. W. 1999, ApJ, 523, 432

Strong, K. T., Claflin, E. S., Lemen, J. R., \& Linford, G. A. 1988, Adv. Space Res., 8, 167

Sylwester, J., Lemen, J. R., \& Mewe, R. 1984, Nature, 310, 665

Tripathi, D., Klimchuk, J. A., \& Mason, H. E. 2011, ApJ, 740, 111

Tsuneta, S., Acton, L., Bruner, M., et al. 1991, Sol. Phys., 136, 37

Veck, N. J., \& Parkinson, J. H. 1981, MNRAS, 197, 41

von Steiger, R., Schwadron, N. A., Fisk, L. A., et al. 2000, J. Geophys. Res., 105,27217

von Steiger, R., Zurbuchen, T. H., \& McComas, D. J. 2010, Geophys. Res. Lett., 37, 22101

Warren, H. P., Winebarger, A. R., \& Brooks, D. H. 2012, ApJ, submitted

Weber, M. A., DeLuca, E. E., Golub, L., \& Sette, A. L. 2004, in IAU Symp. 223, Multi-Wavelength Investigations of Solar Activity, ed. A. V. Stepanov, E. E. Benevolenskaya, \& A. G. Kosovichev (Cambridge: Cambridge Univ. Press), 321

White, S. M., Thomas, R. J., Brosius, J. W., \& Kundu, M. R. 2000, ApJ, 534, 203

Widing, K. G., \& Feldman, U. 1989, ApJ, 344, 1046

Winebarger, A. R., Schmelz, J. T., Warren, H. P., Saar, S. H., \& Kashyap, V. L. 2011, ApJ, 740, 2

Zhang, J., Kundu, M. R., White, S. M., Dere, K. P., \& Newmark, J. S. 2001, ApJ, 561,396 